\title{
From Jacobi off-shell currents to integral relations
}

\author{
José Llanes Jurado, Germán Rodrigo and William J. Torres Bobadilla \\ Instituto de Física Corpuscular, \\ Universitat de València - Consejo Superior de Investigaciones Científicas, \\ Parc Cientific, E-46980 Paterna, Valencia, Spain \\ E-mail: jollaju@alumni.uv.es, german.rodrigo@csic.es, \\ william.torres@ific.uv.es
}

ABSTRACT: In this paper, we study off-shell currents built from the Jacobi identity of the kinematic numerators of $g g \rightarrow X$ with $X=s s, q \bar{q}, g g$. We find that these currents can be schematically written in terms of three-point interaction Feynman rules. This representation allows for a straightforward understanding of the Colour-Kinematics duality as well as for the construction of the building blocks for the generation of higher-multiplicity tree-level and multi-loop numerators. We also provide one-loop integral relations through the Loop-Tree duality formalism with potential applications and advantages for the computation of relevant physical processes at the Large Hadron Collider. We illustrate these integral relations with the explicit examples of QCD one-loop numerators of $g g \rightarrow s s$.

KeYwords: NLO Computations, QCD Phenomenology

ARXIV EPRINT: 1710.11010 


\section{Contents}

1 Introduction 1

2 Colour-kinematics duality 3

3 Jacobi off-shell currents from the colour-kinematics duality 4

$3.1 g g \rightarrow s s \quad 5$

$3.2 \quad g g \rightarrow q \bar{q} \quad 6$

$3.3 \quad g g \rightarrow g g \quad 6$

4 Colour-kinematics duality for multi-leg amplitudes $\quad 7$

4.1 Momentum decomposition in terms of on-shell momenta $\quad 7$

4.2 Construction of numerators from Jacobi off-shell currents 8

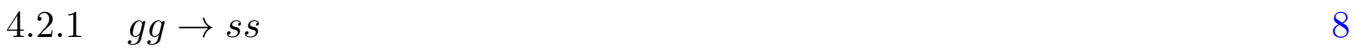

$4.2 .2 \quad g g \rightarrow q \bar{q} \quad 9$

4.2.3 $g g \rightarrow g g \quad 9$

5 Colour-kinematics duality for $2 \rightarrow 2$ processes at one-loop 11

$\begin{array}{lll}5.1 & \text { Integral relations } & 11\end{array}$

$5.2 g g \rightarrow s s$ at one-loop 13

6 Conclusions 16

$\begin{array}{ll}\text { A Three-point interaction Feynman rules } & 17\end{array}$

\begin{tabular}{ll} 
B Projectors & 17 \\
\hline
\end{tabular}

\section{Introduction}

The Large Hadron Collider (LHC) programme demands us to refine our understanding in the calculation of observables in High Energy Physics. The scattering amplitudes are the backbone of theoretical predictions. They, besides of having practical applications in particle physics, also have a mathematical elegance, whose properties have allowed to develop new techniques to perform calculations that a while ago were very cumbersome.

Nevertheless, apart of providing theoretical predictions to phenomenological observables with the use of these amplitudes, we can use their formal properties. These properties are in principle hidden at the Lagrangian level but visible in the scattering amplitudes. In particular, QCD scattering amplitudes can be colour decomposed or simply split into two pieces, one containing information of the colour structure and other one taking care of kinematic variables [1-5]. The latter is often called primitive amplitude. An n-point colour-dressed tree-level amplitude generates $n$ ! primitive amplitudes, although, due to cyclic invariance the number of independent primitive amplitudes is reduced to $(n-1)$ !. In the same manner, with a proper choice of the basis for the colour structure $[6,7]$, the Kleiss-Kuijf relations [8] reduce the number of independent primitive amplitudes to $(n-2)$ !. 


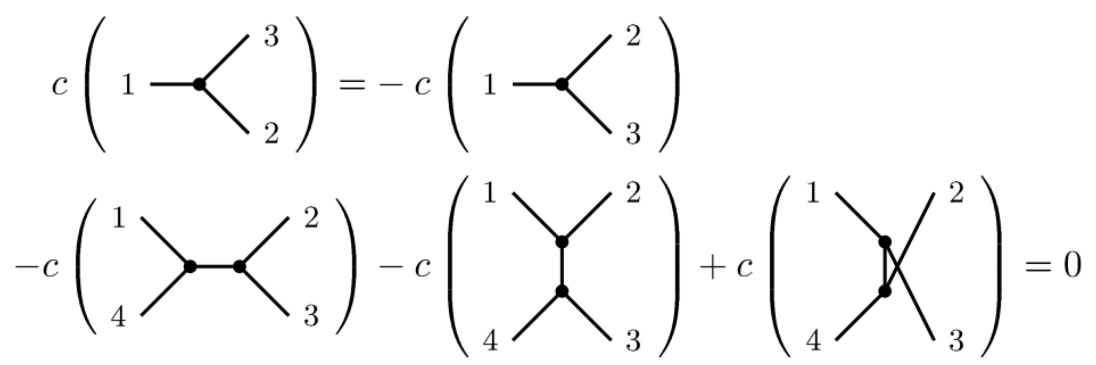

Figure 1. Colour anti-symmetry relation and Jacobi identity.

Remarkably, it has been found by Bern, Carrasco and Johansson (BCJ) [9, 10] that scattering amplitudes in gauge theories satisfy a colour-kinematic dual representation. This representation states that the kinematic part of numerators of Feynman diagrams obeys Jacobi identities and anti-symmetric relations, similar to the corresponding structure constants and group generators of the colour algebra, see figure 1. Therefore, the rearrangement of numerators, according to these identities, leads to a set of relations between primitive amplitudes, called BCJ relations, reducing further the number of independent primitive amplitudes to $(n-3)$ !.

Several calculations have been done with the use of the colour-kinematics duality (CKD), going from supersymmetric theories, up to five-loop level [10-20], to non-supersymmetric ones, up to two-loop level [21-23]. Similarly, new relations at oneloop level have been found with a clever use of the BCJ relations with string theory [24-28] and unitarity based methods $[29,30]$.

In this paper we follow a diagrammatic approach to construct compact expressions for off-shell currents built from the Jacobi identity of kinematic numerators. First, we focus on the $2 \rightarrow 2$ processes considered in ref. [31] by one of the current authors, $g g \rightarrow X$ with $X=s s, q \bar{q}, g g$, finding that CKD can be cast into a systematic representation, whose shape relies on three-point interactions. These off-shell currents vanish if the external particles are set on-shell, thus satisfying CKD. A pictorial representation is given in terms of Feynman rules. This compact representation of off-shell currents allows for a straightforward generation of CKD tree-level amplitudes, by following the algorithms based on gauge transformations [31-34].

Later, we investigate the properties of these off-shell currents when they are embedded in a richer topology, namely higher-multiplicity or multi-loop scattering amplitudes. It turns out that with a proper decomposition of the four off-shell momenta in on-shell massless ones we find a simple structure that allows to write objects built from CKD off-shell currents in terms of at most two squared off-shell momenta. We remark that this decomposition, showing the full off-shell dependence, does not have additional contributions of squared momenta.

As a byproduct of the off-shell decomposition at one-loop level, we show that new relations among Feynman integrals with the same number of propagators emerge. In order to extract these identities, we use the Loop-Tree duality (LTD) formalism [35-39]. To 
this end, we consider, for illustrative reasons, the one-loop process of $g g \rightarrow s s$, whose numerators are built from Jacobi off-shell currents. We note that numerators with higher rank in the loop momentum are written in terms of lower ones.

Algebraic manipulations have been carried out by using the MATHEMATICA packages Feynarts [40] and FeynCalC [41, 42].

\section{Colour-kinematics duality}

In this section, we set up the notation and normalisation used throughout this paper. Let us first consider an $m$-point tree-level amplitude

$$
\mathcal{A}_{m}^{\text {tree }}(1,2, \ldots, m)=\sum_{i=1}^{N} \frac{c_{i} n_{i}}{D_{i}} \quad D_{i}=\prod_{\alpha_{i}} s_{\alpha_{i}},
$$

where the sum runs over all diagrams $i$ with only cubic vertices, $c_{i}$ are the colour factors, $n_{i}$ the kinematic numerators, and $D_{i}$ collect the denominators of all internal propagators. As we shall see in the next section, contact terms are absorbed into cubic diagrams once they are replaced with numerator factors cancelling propagators, i.e., $s_{\alpha} / s_{\alpha}$ and assigning their contribution to the proper diagram according to the colour factor.

The main property of the colour factors is that they satisfy the Jacobi identities

$$
\begin{aligned}
-\tilde{f}^{a_{1} a_{2} x} \tilde{f}^{a_{3} a_{4} x}-\tilde{f}^{a_{1} a_{4} x} \tilde{f}^{a_{2} a_{3} x}+\tilde{f}^{a_{1} a_{3} x} \tilde{f}^{a_{2} a_{4} x} & =0, \\
-\tilde{f}^{a_{1} a_{2} x} T^{x}-T^{a_{1}} T^{a_{2}}+T^{a_{2}} T^{a_{1}} & =0,
\end{aligned}
$$

where we have adopted the normalisation $\tilde{f}^{a b c}=\operatorname{Tr}\left(\left[T^{a}, T^{b}\right] T^{c}\right)=i \sqrt{2} f^{a b c}$ and $T^{a}=$ $\sqrt{2} t^{a}$, with $f^{a b c}$ and $t^{a}$ the standard structure constants and generators of $\mathrm{SU}(\mathrm{N})$, to avoid prefactors in the next calculations.

Furthermore, for any $m$-point amplitude, we can always find three colour factors built from (2.2), say

$$
c_{i}=\ldots \tilde{f}^{a_{1} a_{2} x} \tilde{f}^{a_{3} a_{4} x} \ldots, \quad c_{j}=\ldots \tilde{f}^{a_{1} a_{4} x} \tilde{f}^{a_{2} a_{3} x} \ldots, \quad c_{k}=\ldots \tilde{f}^{a_{1} a_{3} x} \tilde{f}^{a_{2} a_{4} x} \ldots,
$$

where the '...' state for common terms in the three colour factors. Therefore, the Jacobi identity takes the form

$$
-c_{i}-c_{j}+c_{k}=0
$$

Since colour factors and kinematic numerators satisfy anti-symmetry relations under a swapping of legs, $c_{j} \rightarrow-c_{j} \Rightarrow n_{j} \rightarrow-n_{j}$, we promote (2.4) to be dual in the kinematic sector,

$$
-n_{i}-n_{j}+n_{k}
$$

This relation between colour factors and kinematic numerators is referred to as ColourKinematics duality (CKD). CKD is always satisfied for $2 \rightarrow 2$ processes at tree-level. However, its generalisation for processes at higher-multiplicity or multi-loop level is not straightforward. In fact, CKD for tree-level amplitudes can always be recovered by rearranging numerators but at multi-loop level it has not been proven yet remaining as a conjecture [43]. 
a)

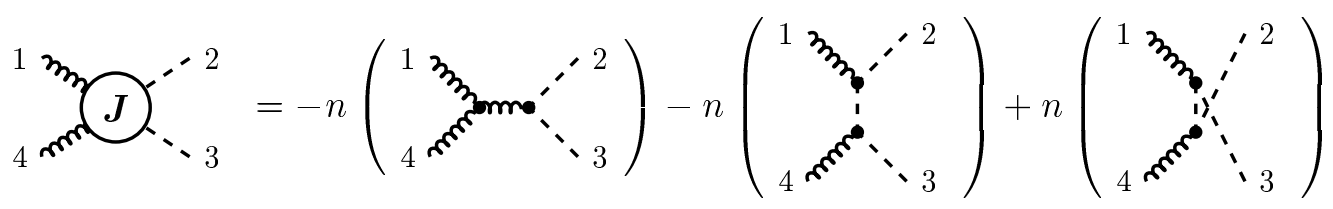

b)

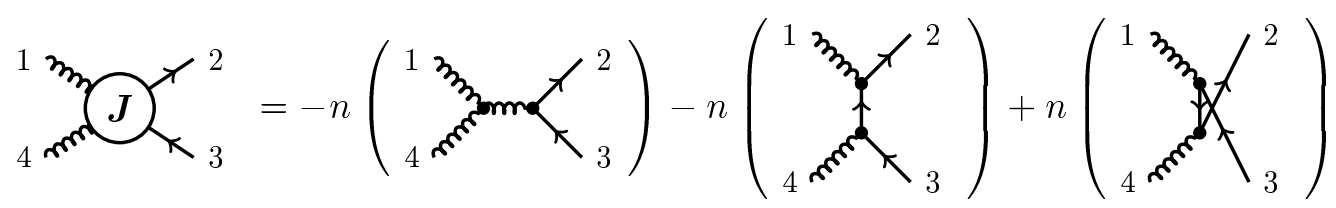

c)

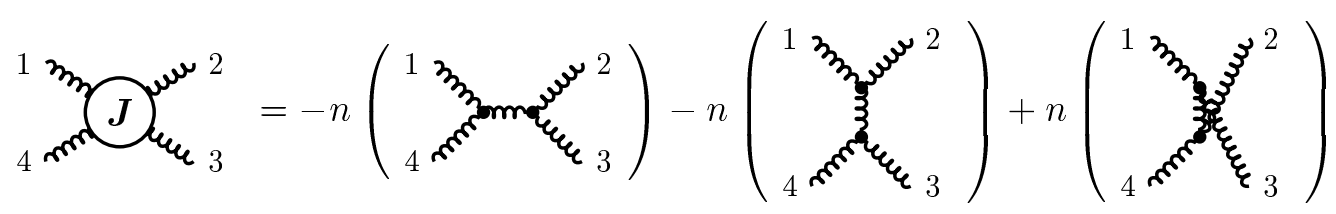

Figure 2. Jacobi combination for $g g \rightarrow X$ with $X=s s, q \bar{q}, g g$.

\section{Jacobi off-shell currents from the colour-kinematics duality}

In this section, we compute off-shell currents built from the Jacobi-like combination of numerators. We recap the diagrammatic approach of ref. [31], used by one of the current authors. We find that this combination of numerators for the processes $g g \rightarrow X$, with $X=s s, q \bar{q}, g g$ can always be schematically represented in terms of three-point interaction Feynman rules.

These off-shell currents are computed in the axial gauge, in which the polarisation tensor of the gluon propagator is written as

$$
\Pi^{\alpha \beta}\left(p_{i}, q\right)=-g^{\alpha \beta}+\frac{p_{i}^{\alpha} q^{\beta}+p_{i}^{\beta} q^{\alpha}}{p_{i} \cdot q},
$$

where $p_{i}$ is the momentum of the internal (or off-shell) gluon and $q$ its gauge reference momentum, such that $q^{2}=0$ and $p_{i} \cdot q \neq 0$. Throughout this paper the reference momentum $q$ is chosen to be the same for all internal gluons. Although, this choice of gauge does not play any role for $2 \rightarrow 2$ processes at tree-level it allows for simplifications when considering processes with higher-multiplicity at tree or multi-loop level, as we shall observe in section 4 .

CKD is studied for the processes depicted in figure 2. We consider quark (anti-quark) living in the fundamental (anti-fundamental) representation of SU(N), while scalars and gluons are in the adjoint one.

In figure $2, n(\ldots)$ is understood as the numerator of the Feynman diagram, in which scalar propagators, $1 / p_{i}^{2}$, have been removed. Nevertheless, contributions of $1 /\left(p_{i} \cdot q\right)$ are retained in order to work out with same dimensions. The four-point interactions, or simply contact terms, ssgg and gggg have been rewritten in terms of cubic diagrams only. Thus, a numerator containing one contact term can be expressed as

$$
n_{4}=s_{\alpha_{i}} n_{4: i} c_{i}+s_{\alpha_{j}} n_{4: j} c_{j}+s_{\alpha_{k}} n_{4: k} c_{k} .
$$


We compute the Jacobi off-shell currents for the processes $g g \rightarrow s s, g g \rightarrow q \bar{q}$ and $g g \rightarrow g g$ in axial gauge. In order to elucidate the dependence on the gauge, we split the propagator (3.1) into Feynman and covariant parts, i.e.,

$$
\Pi^{\mu \nu}\left(p_{i}, q\right)=\Pi_{\mathrm{Fey}}^{\mu \nu}+\Pi_{\mathrm{Ax}}^{\mu \nu}\left(p_{i}, q\right)
$$

with $\Pi_{\text {Fey }}^{\mu \nu}=-g^{\mu \nu}$ and $\Pi_{\mathrm{Ax}}^{\mu \nu}\left(p_{i}, q\right)=\frac{p_{i}^{\mu} q^{\nu}+p_{i}^{\nu} q^{\mu}}{p_{i} \cdot q}$. This splitting allows us to write the Jacobi off-shell currents as

$$
J_{\mathrm{x}}^{\mu_{1} \ldots \mu_{4}}=J_{\mathrm{x}-\mathrm{Fey}}^{\mu_{1} \ldots \mu_{4}}+J_{\mathrm{x}-\mathrm{Ax}}^{\mu_{1} \ldots \mu_{4}}, \quad \text { with } \mathrm{x}=\mathrm{s}, \mathrm{q}, \mathrm{g} .
$$

In order to provide a simple and compact representation for the Jacobi off-shell currents, we use Feynman-like graphs to write them schematically in terms of three-point interaction Feynman rules. Nevertheless, these expressions do not always obey the usual properties of the Feynman rules. In fact, the momenta of the three-point interactions are not related by momentum conservation. Furthermore, these interactions could not correspond to a physical process as we shall see for the process $g g \rightarrow q \bar{q}$, that includes the insertion of an interaction between one quark and two gluons.

In the following, we list the Jacobi off-shell currents.

\section{$3.1 \quad g g \rightarrow s s$}

We start considering the process $g g \rightarrow s s$ with gluons and scalars in the adjoint representation. The Jacobi identity for kinematic numerator takes the form,

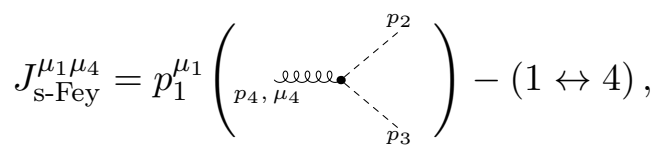

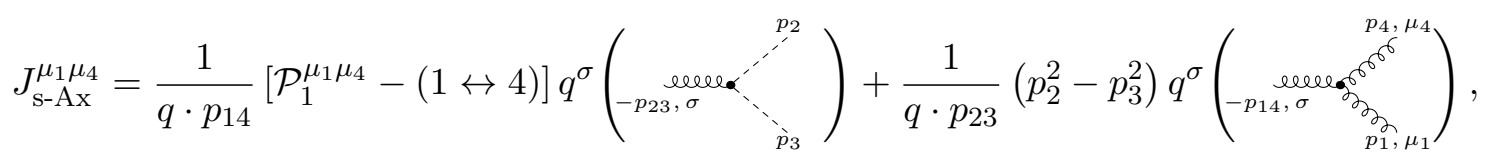

where $p_{i j}^{\alpha} \equiv\left(p_{i}+p_{j}\right)^{\alpha}$ and $\mathcal{P}_{i}^{\mu_{i} \mu_{j}} \equiv p_{i}^{\mu_{i}} p_{i}^{\mu_{j}}-p_{i}^{2} g^{\mu_{i} \mu_{j}}$. The Feynman rules for the three-point interaction vertices are defined in appendix A.

In eq. (3.5), contributions to $J_{\text {s-Fey }}$ do not obey momentum conservation. It only follows the structure of the Feynman rule for gss, eq. (A.1). On the other hand, contributions to $J_{\mathrm{s}-\mathrm{Ax}}$ do obey momentum conservation.

We remark that contributions to $J_{\text {s-Fey }}$ come from the three diagrams of figure 2.a, whereas $J_{\text {s-Ax }}$ gets only one contribution from the diagram with an internal gluon. Additionally, we note that CKD for the former is satisfied when the external gluons are set on-shell, or simply ask for transversality condition, $\varepsilon\left(p_{i}\right) \cdot p_{i}=0$. In the latter, both gluons and scalars have to be set on-shell, $p_{i}^{2}=0$. 


\section{$3.2 \quad g g \rightarrow q \bar{q}$}

We now consider the process $g g \rightarrow q \bar{q}$ with quark (anti-quark) in the fundamental representation. The Jacobi off-shell current $J_{\mathrm{q}}$ takes the form,

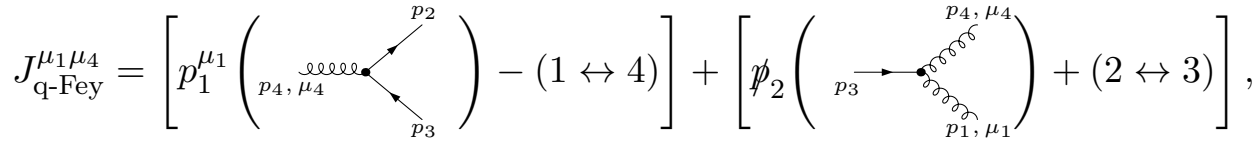

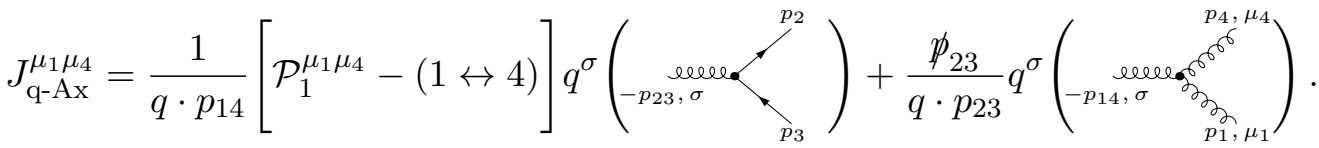

In eq. (3.6) we have included an additional interaction between one quark and two gluons, (A.3). This Feynman rule does not obey momentum conservation and does not have any physical meaning. It nevertheless allows for a universality in the decomposition of the off-shell current generated from CKD.

Similar to $J_{\mathrm{s}}$, contributions to $J_{\mathrm{q}-\mathrm{Fey}}$ come from the three diagrams of figure 2.b, whereas, $J_{\mathrm{q}-\mathrm{Ax}}$ gets contribution from the diagram with an internal gluon. We also see that in order to recover CKD the four particles have to be set on-shell. For this case, apart from asking for transversality conditions of external gluons we also need external quark (anti-quark) to satisfy Dirac equations, $\not p_{2} \bar{u}\left(p_{2}\right)=v\left(p_{3}\right) \not p_{3}=0$.

The exchanging $(2 \leftrightarrow 3)$ in eq. (3.6) takes also care of the Dirac indices. Hence, any expression containing gamma matrices should intrinsically be read as follows,

$$
\not p_{2}\left[\gamma^{\mu_{1}}, \gamma^{\mu_{4}}\right] \equiv\left(\not p_{2}\right)_{i_{2} \bar{k}}\left[\gamma^{\mu_{1}}, \gamma^{\mu_{4}}\right]_{k \bar{\jmath}_{3}}
$$

therefore,

$$
\not p_{2}\left[\gamma^{\mu_{1}}, \gamma^{\mu_{4}}\right] \underset{2 \leftrightarrow 3}{\longrightarrow}\left[\gamma^{\mu_{4}}, \gamma^{\mu_{1}}\right] \not p_{3}
$$

\section{$3.3 \quad g g \rightarrow g g$}

As last application, we consider the process of $g g \rightarrow g g$. Since there is no mixing between different kind of particles, we end up with the most compact and symmetric expression for the Jacobi off-shell current $J_{\mathrm{g}}$,

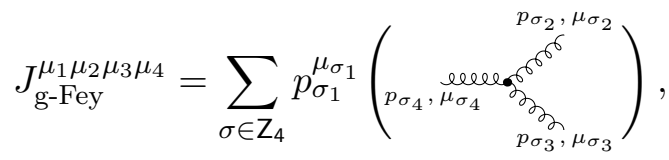

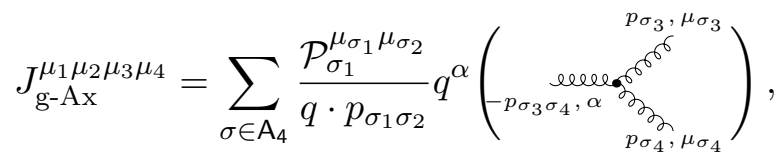

where the sums run over the all the permutations of the cyclic $\left(Z_{4}\right)$ and alternative $\left(A_{4}\right)$ groups, respectively, whose elements are represented by $\sigma_{i}$. 
As in the previous cases, $J_{\text {g-Fey }}$ comes from the three diagrams of figure 2.c and their contributions in terms of Feynman rules do not obey momentum conservation as they do for the ones in $J_{\mathrm{g} \text {-Ax }}$. Furthermore, we observe a similar pattern between $J_{s}$ and $J_{g}$. In fact, the former can be easily recovered from the latter by setting $p_{i}^{\mu_{2}}=p_{i}^{\mu_{3}}=0$ and $g^{\mu_{2} \mu_{3}}=1$, for $i=1, \ldots, 4$.

From $J_{\mathrm{s}, \mathrm{q}, \mathrm{g}}$, eq.s (3.5), (3.6) and (3.9), we observe that Jacobi off-shell currents can systematically be cast into objects whose structure follow the three-point interaction Feynman rules. We remark that all $J_{\mathrm{x} \text {-Fey }}$ are obtained from the Jacobi identity of three kinematic numerators of eq. (2.5) and their structure, after algebraic manipulations, is always written as Feynman rules where momentum conservation is not preserved. Likewise, CKD is straightforwardly recovered when the four particles in the off-shell currents are set on-shell. On the other hand, the Feynman rules appearing in all $J_{\mathrm{x}-\mathrm{Ax}}$ do obey momentum conservation and the way how CKD is satisfied is individually at the level of diagrams. It is indeed for this reason that the single diagram with an internal gluon for $g g \rightarrow s s$ and $g g \rightarrow q \bar{q}$ vanishes by its own when the four particles are set on-shell. In fact, contributions from the covariant part of the polarisation tensor of the gluon propagator (3.1), $q^{\mu} p^{\nu}+q^{\nu} p^{\mu}$, manifest the same pattern,

$$
p_{\sigma_{1}}^{\mu_{\sigma_{1}}} p_{\sigma_{1}}^{\mu_{\sigma_{2}}}-p_{\sigma_{1}}^{2} g^{\mu_{\sigma_{1}} \mu_{\sigma_{2}}}
$$

which vanishes when it is contracted with $\varepsilon^{\mu_{\sigma_{i}}}\left(p_{\sigma_{i}}\right)$ and on-shellness of $p_{\sigma_{i}}$ is imposed, $p_{\sigma_{i}}^{2}=0$.

\section{Colour-kinematics duality for multi-leg amplitudes}

In section 3 we constructed off-shell currents from the Jacobi identity of numerators. Hence, in order to understand their behaviour when they are embedded in a richer topology, trees with higher-multiplicity or multi-loop level, we plug external wave functions that do not necessary have to be on-shell. We find that numerators built from Jacobi off-shell currents, $J_{\mathrm{s}, \mathrm{q}, \mathrm{g}}$, are always written in terms of at most the product of two squared momenta. Being these momenta the ones attached to the off-shell current.

\subsection{Momentum decomposition in terms of on-shell momenta}

Let us decompose an off-shell momentum $p_{i}$ into two on-shell momenta,

$$
p_{i}^{\alpha}=r_{i}^{\alpha}+\frac{p_{i}^{2}}{2 q \cdot r_{i}} q^{\alpha}
$$

where $r_{i}$ and $q$ are massless momenta, $r_{i}^{2}=q^{2}=0$, with the condition $r_{i} \cdot q \neq 0$. For the purpose of our calculations, $q$ is chosen to be same as the reference momenta of the gluon propagator in the axial gauge (3.1).

Hence, completeness relations for polarisation vectors, in axial gauge, and spinors take the form

$$
\begin{aligned}
& \sum_{\lambda=1}^{d_{s}-2} \varepsilon_{\lambda\left(d_{s}\right)}^{\alpha}\left(p_{i}\right) \varepsilon_{\lambda\left(d_{s}\right)}^{* \beta}\left(p_{i}\right)=-g^{\alpha \beta}+\frac{r_{i}^{\alpha} q^{\beta}+r_{i}^{\beta} q^{\alpha}}{r_{i} \cdot q}+\frac{p_{i}^{2}}{\left(r_{i} \cdot q\right)^{2}} q^{\alpha} q^{\beta}, \\
& \sum_{\lambda=1}^{2^{\left(d_{s}-2\right) / 2}} u_{\lambda\left(d_{s}\right)}\left(p_{i}\right) \bar{u}_{\lambda\left(d_{s}\right)}\left(p_{i}\right)=\psi_{i}+\frac{p_{i}^{2}}{2 r_{i} \cdot q} \not \mu .
\end{aligned}
$$


The completeness relations (4.2) and (4.3) distinguish between on- and off-shell quantities. The on-shell ones account for the numerator of gluon and fermion propagators, whereas the off-shell ones take care of contributions coming from $p_{i}^{2}$ only. Therefore, these completeness relations can be cast into,

$$
\begin{aligned}
\sum_{\lambda=1}^{d_{s}-2} \varepsilon_{\lambda\left(d_{s}\right)}^{\alpha}\left(p_{i}\right) \varepsilon_{\lambda\left(d_{s}\right)}^{* \beta}\left(p_{i}\right) & =\sum_{\lambda_{i}=1}^{d_{s}-2} \varepsilon_{i}^{\alpha} \varepsilon_{i}^{* \beta}+\frac{p_{i}^{2}}{\left(r_{i} \cdot q\right)^{2}} q^{\alpha} q^{\beta}, \\
\sum_{\lambda=1}^{2^{\left(d_{s}-2\right) / 2}} u_{\lambda\left(d_{s}\right)}\left(p_{i}\right) \bar{u}_{\lambda\left(d_{s}\right)}\left(p_{i}\right) & =\sum_{\lambda_{i}=1}^{2^{\left(d_{s}-2\right) / 2}} u_{i} \bar{u}_{i}+\frac{p_{i}^{2}}{2 r_{i} \cdot q} q .
\end{aligned}
$$

Here and in the following we use the abbreviations

$$
\varepsilon_{i}^{\mu} \equiv \varepsilon_{\lambda_{i}\left(d_{s}\right)}^{\mu}\left(r_{i}\right), \quad \bar{u}_{i} \equiv \bar{u}_{\lambda_{i}\left(d_{s}\right)}\left(r_{i}\right), \quad v_{i} \equiv v_{\lambda_{i}\left(d_{s}\right)}\left(r_{i}\right)
$$

As mentioned above, we have chosen $q$ to be the same for all off-shell momenta. This is done to remove, as much as possible, redundant terms in the following calculations. Thus, besides the on-shell conditions $r_{i}$ satisfy, $\varepsilon_{i} \cdot r_{i}=0$ and $\bar{u}_{i} \eta_{i}=\not_{i} v_{i}=0$, we also have, as a consequence of decomposition (4.1), $\varepsilon_{i} \cdot q=\varepsilon_{i} \cdot p_{i}=0$.

\subsection{Construction of numerators from Jacobi off-shell currents}

In order to study the behaviour of numerators built from the Jacobi off-shell currents, we consider

$$
\begin{aligned}
N_{\mathrm{s}} & =N_{\mathrm{s} \mu_{1} \mu_{4}} X^{\mu_{1} \mu_{4}}, & N_{\mathrm{s} \mu_{1} \mu_{4}} & =J_{\mathrm{s}}^{\nu_{1} \nu_{4}} \Pi_{\mu_{1} \nu_{1}}\left(p_{1}, q\right) \Pi_{\mu_{4} \nu_{4}}\left(p_{4}, q\right), \\
N_{\mathrm{q}} & =N_{\mathrm{q} \mu_{1} \mu_{4}} X^{\mu_{1} \mu_{4}}, & N_{\mathrm{q} \mu_{1} \mu_{4}} & =\not p_{2} J_{\mathrm{q}}^{\nu_{1} \nu_{4}} \not p_{3} \Pi_{\mu_{1} \nu_{1}}\left(p_{1}, q\right) \Pi_{\mu_{4} \nu_{4}}\left(p_{4}, q\right), \\
N_{\mathrm{g}} & =N_{\mathrm{g} \mu_{1} \ldots \mu_{4}} X^{\mu_{1} \ldots \mu_{4}}, & N_{\mathrm{g} \mu_{1} \ldots \mu_{4}} & =J_{\mathrm{g}}^{\nu_{1} \ldots \nu_{4}} \Pi_{\mu_{1} \nu_{1}}\left(p_{1}, q\right) \ldots \Pi_{\mu_{4} \nu_{4}}\left(p_{4}, q\right) .
\end{aligned}
$$

The tensors $X$ carry the information related to the kinematic part where the off-shell currents $J_{\mathrm{s}, \mathrm{q}, \mathrm{g}}$ are embedded. They can generate either tree-level or multi-loop numerators.

In the case that any of the gluons attached to the Jacobi off-shell current is set onshell, the polarisation tensor of the gluon should be substituted by the corresponding polarisation vector. Analogously for quarks, the $\not p_{i}$ in eq. (4.6b) should be substituted by the corresponding spinor.

Let us begin by defining a shorthand notation for the definition of the numerators

$$
\mathcal{E}_{i j}^{\nu_{i} \nu_{j}} \equiv \sum_{\lambda_{i}, \lambda_{j}=1}^{d_{s}-2} \varepsilon_{i}^{\nu_{i}} \varepsilon_{j}^{\nu_{j}}, \quad \mathcal{Q}_{i}^{\nu_{i} \nu_{j}} \equiv \sum_{\lambda_{j}=1}^{d_{s}-2} q^{\nu_{i}} \varepsilon^{\nu_{j}}, \quad \mathfrak{q}^{\nu_{i} \nu_{j}} \equiv q^{\nu_{i}} q^{\nu_{j}}, \quad \boldsymbol{\eta}_{i} \equiv \sum_{\lambda_{i}=1}^{2^{\left(d_{s}-2\right) / 2}} u_{i} \bar{u}_{i} .
$$

In the following, we list the numerators (4.6) built from the Jacobi off-shell currents.

\subsection{1 $g g \rightarrow s s$}

We start considering the generic structure of numerators built from the Jacobi off-shell current $J_{s}$,

$$
N_{\mathrm{s}}^{\mu_{1} \mu_{4}}=\sum_{i, j=1,4} \epsilon_{i j} p_{i}^{2}\left(A_{i j} \mathcal{E}_{i j}^{\mu_{i j} \mu_{j}}+B_{i j} \mathcal{Q}_{i}^{\mu_{i} \mu_{j}}+C_{i j} p_{j}^{2} \mathfrak{q}^{\mu_{i} \mu_{j}}\right)+\left(p_{2}^{2}-p_{3}^{2}\right) \tilde{A}_{14} \mathcal{E}_{14}^{\mu_{1} \mu_{4}}
$$


with $\epsilon_{i j}$ the Levi-Civita tensor with the signature $\epsilon_{14}=+1$ and

$$
\begin{array}{ll}
A_{i j}=\frac{q \cdot\left(r_{2}-r_{3}\right)}{q \cdot r_{23}} \varepsilon_{i} \cdot \varepsilon_{j}, & B_{i j}=-\frac{1}{q \cdot r_{i}}\left[\frac{q \cdot\left(r_{2}-r_{3}\right)}{q \cdot r_{23}} \varepsilon_{j} \cdot r_{i}+\varepsilon_{j} \cdot\left(r_{2}-r_{3}\right)\right], \\
\tilde{A}_{14}=\frac{q \cdot\left(r_{1}-r_{4}\right)}{q \cdot r_{14}} \varepsilon_{1} \cdot \varepsilon_{4}, & C_{i j}=\frac{q \cdot\left(r_{2}-r_{3}\right) q \cdot\left(r_{i}-r_{j}\right)}{\left(q \cdot r_{1}\right)^{2}\left(q \cdot r_{4}\right)^{2}}
\end{array}
$$

From eq. (4.8), we do not get terms proportional to $p_{i}^{2} p_{j}^{2}$ with $i=1, \ldots, 4, j=2,3$ and $i \neq j$, because $q \cdot\left(p_{4}-p_{1}\right)=q \cdot\left(r_{4}-r_{1}\right)$.

\subsection{2 $\quad g g \rightarrow q \bar{q}$}

We now turn our attention to numerators built from the Jacobi off-shell current $J_{q}$,

$$
\begin{aligned}
& N_{\mathrm{q}}^{\nu_{1} \nu_{4}}=\left\{p _ { 1 } ^ { 2 } \left[\frac{\gamma_{2} q \psi_{3}}{q \cdot r_{23}}\left(\varepsilon_{1} \cdot \varepsilon_{4} \mathcal{E}_{14}^{\nu_{1} \nu_{4}}-\frac{\varepsilon_{4} \cdot r_{1}}{q \cdot r_{1}} \mathcal{Q}_{4}^{\nu_{1} \nu_{4}}+p_{4}^{2} \frac{q \cdot r_{23} q \cdot\left(r_{1}-r_{4}\right)}{\left(q \cdot r_{1}\right)^{2}\left(q \cdot r_{4}\right)^{2}} \mathfrak{q}^{\nu_{1} \nu_{4}}\right)\right.\right. \\
& \left.\left.+\frac{1}{q \cdot r_{1}}\left(\nvdash_{2} \ddagger_{4} \psi_{3}+p_{2}^{2} \frac{\not \ddagger_{4} \psi_{3}}{2 q \cdot r_{2}}+p_{3}^{2} \frac{\psi_{2} \ddagger_{4} q}{2 q \cdot r_{3}}\right) \mathcal{Q}_{4}^{\nu_{1} \nu_{4}}\right]-(1 \leftrightarrow 4)\right\} \\
& +\left\{\frac { p _ { 2 } ^ { 2 } } { 2 } \left[\notin_{1} \oiint_{4} p_{3} \mathcal{E}_{14}^{\nu_{1} \nu_{4}}+\left[\oint_{1}, q\right] \frac{\nvdash_{3}}{q \cdot r_{4}} p_{4}^{2} \mathcal{Q}_{1}^{\nu_{4} \nu_{1}}\right.\right. \\
& \left.\left.-2 \varepsilon_{1} \cdot \varepsilon_{4} q \cdot r_{1}\left(\frac{r_{3}}{q \cdot r_{23}}+p_{3}^{2} \frac{q}{4\left(q \cdot r_{2}\right)\left(q \cdot r_{3}\right)}\right) \mathcal{E}_{14}^{\nu_{1} \nu_{4}}-(1 \leftrightarrow 4)\right]+(2 \leftrightarrow 3)\right\} .
\end{aligned}
$$

Unlike the case of the $N_{\mathrm{s}}$, we do not get a simple structure. This behaviour is because we are basically mixing two kind of particles, gluons in the adjoint and quarks in the fundamental representation. The former are governed by Lorentz indices whereas the latter accounts for Dirac ones. However, we see that numerators $N_{\mathrm{q}}$ can generically be decomposed as

$$
N_{\mathrm{q}}=\sum_{i=1}^{4} c_{i} p_{i}^{2}+\sum_{\substack{i, j=1 \\ i \neq j}}^{4} c_{i j} p_{i}^{2} p_{j}^{2}
$$

with $c$, coefficients depending on the kinematics where the off-shell current $J_{q}$ is embedded in. On top of it, we note a difference with numerators $N_{\mathrm{s}}$, since now all powers of $p_{i}^{2} p_{j}^{2}$, for $i, j=1, \ldots, 4$ with $i \neq j$, are present.

\subsection{3 $\quad g g \rightarrow g g$}

As last application, we now consider numerators built from the Jacobi off-shell current $J_{g}$. Since we are working with particles of the same kind, the numerator $N_{\mathrm{g}}$ is compactly expressed as,

$$
N_{\mathrm{g}}^{\nu_{1} \ldots \nu_{4}}=\frac{1}{2} \sum_{i, j, k, l=1}^{4} \epsilon_{i j k l} p_{i}^{2}\left(A_{i j k l} \mathcal{E}_{i j}^{\nu_{i} \nu_{j}} \mathcal{E}_{k l}^{\nu_{k} \nu_{l}}+B_{i j k l} \mathcal{E}_{j k}^{\nu_{j} \nu_{k}} \mathcal{Q}_{l}^{\nu_{i} \nu_{l}}+C_{i j k l} p_{j}^{2} \mathfrak{q}^{\nu_{i} \nu_{j}} \mathcal{E}_{k l}^{\nu_{k} \nu_{l}}\right)
$$


with $\epsilon_{i j k l}$ the Levi-Civita tensor with the signature $\epsilon_{1234}=+1$ and

$$
\begin{aligned}
A_{i j k l} & =\frac{q \cdot\left(r_{k}-r_{l}\right)}{q \cdot\left(r_{k}+r_{l}\right)} \varepsilon_{i} \cdot \varepsilon_{j} \varepsilon_{k} \cdot \varepsilon_{l}, \\
B_{i j k l} & =-\frac{\varepsilon_{j} \cdot \varepsilon_{k}}{q \cdot r_{i}}\left[\frac{q \cdot\left(r_{j}-r_{k}\right)}{q \cdot\left(r_{j}+r_{k}\right)} \varepsilon_{l} \cdot r_{i}+\varepsilon_{l} \cdot\left(r_{j}-r_{k}\right)\right], \\
C_{i j k l} & =\frac{q \cdot\left(r_{i}-r_{j}\right) q \cdot\left(r_{k}-r_{l}\right)}{\left(q \cdot r_{i}\right)^{2}\left(q \cdot r_{j}\right)^{2}} \varepsilon_{k} \cdot \varepsilon_{l} .
\end{aligned}
$$

We also observe that numerators built from $J_{g}$ contain all possible combinations of $p_{i}^{2} p_{j}^{2}$, similarly to $N_{\mathrm{q}}$ in eq. (4.11).

We notice from the above results that, because of the way the off-shell wave-functions are parametrised, there are no terms proportional to $p_{i}^{2} p_{j}^{2} p_{k}^{2} p_{l}^{2}$ or $p_{i}^{2} p_{j}^{2} p_{k}^{2}$. This is indeed due to the choice of a unique reference momentum $q$ in the definition of internal propagators and wave-functions. Therefore, we can claim that any numerator built from the off-shell currents $J_{\mathrm{s}, \mathrm{q}, \mathrm{g}}$ is written in terms of at most the product of two squared momenta, $p_{i}^{2} p_{j}^{2}$. Although this observation was done in [31], it is not completely true for the general case where all reference momenta for the internal gluons are chosen to be different. In fact, after the off-shell momenta are decomposed according to eq. (4.1), with $q=q_{i}$, contributions of the product of three and four squared momenta arise. In particular, scalar products $\varepsilon_{i} \cdot q_{j}$ for $i \neq j$ are non-vanishing anymore.

There are particular cases where CKD can be immediately satisfied for multi-leg processes. This is the case of the three-gluon vertex contributions to $X^{\mu_{1} \ldots \mu_{4}}$, in the Feynman gauge. In fact, if the fouth leg, a gluon, of any Jacobi off-shell current is set off-shell, we can end up with

$$
\begin{gathered}
J_{\text {x-Fey }}^{\mu_{1} \ldots \nu_{45}}\left(p_{1}, p_{2}, p_{3}, p_{45}\right) V_{g g g \nu_{45}}^{\mu_{4} \mu_{5}}\left(-p_{45}, p_{4}, p_{5}\right) \varepsilon_{\mu_{4}}\left(p_{4}\right) \varepsilon_{\mu_{5}}\left(p_{5}\right)=0, \\
J_{\text {x-Fey }}^{\mu_{1} \ldots \nu_{45}}\left(p_{1}, p_{2}, p_{3}, p_{45}\right) \bar{u}\left(p_{4}\right) V_{g q \bar{q} \nu_{45}}\left(-p_{45}, p_{4}, p_{5}\right) u\left(p_{5}\right)=0,
\end{gathered}
$$

with $V_{g g g}$ and $V_{g q \bar{q}}$ the standard three-gluon and quark-anti-quark-gluon vertices.

It turns out that for $J_{\mathrm{x}-\mathrm{Fey}}^{\mu_{1} \ldots \nu_{45}}$, with $\mathrm{x}=\mathrm{s}, \mathrm{g}$, we can generate (up to) eight-point numerators built from these off-shell currents. Whereas, for $J_{\mathrm{q}-\mathrm{Fey}}^{\mu_{1} \ldots \nu_{45}}$ we can do the same for (up to) six-point numerator. We do not recover this kind of identity for the emission of a gluon from a fermion line, since we get, as expected from the above discussion, a term proportional to squared momenta.

We stress that $X$ 's made of three-gluon vertices miss all the contributions from contact terms. Nevertheless, adding these contact terms spoils the generation of a numerator satisfying CKD. This is due to the fact that diagrams containing a contact term have to be slightly modified in order to be consequent with the definition of three-point vertices. Indeed, those diagrams have one propagator less and are naively restored by inserting factors of $p_{i}^{2} / p_{i}^{2}$. The proper insertion of contact terms allows for a generation of numerators satisfying CKD from construction, as was done in ref. [44] for Yang-Mills theories.

In the same manner, when considering theories with particles living in different representations, like gluons and quarks in QCD, it has been shown that the arbitrary procedure 
a) $N_{3}=$
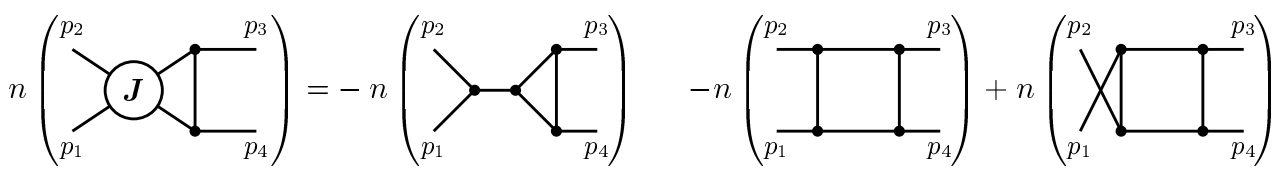

b)

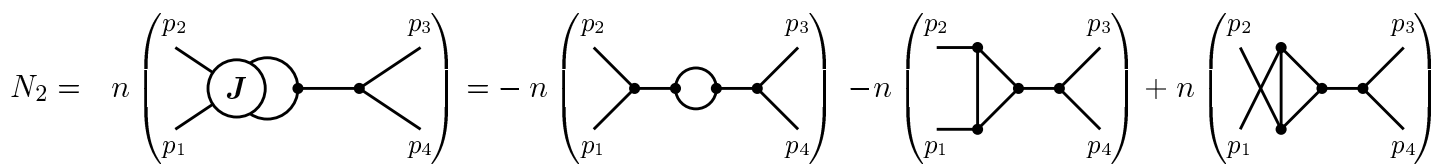

Figure 3. Jacobi combinations for $2 \rightarrow 2$ processes at one-loop.

of rearranging diagrams with contact terms does not allow a systematic generation of numerators satisfying CKD. In particular, ref.s [45-48] discuss the automatic generation of dual numerators. This relation is straightforwardly satisfied when in the generation of the kinematic numerators there are true three-point vertices, i.e. no contact terms. The latter relies on the emission of single gluons. In order to compute processes with mostly quarks one could take advantage of Melia's formalism [45]. This formalism allows us to compute processes with quarks of different flavours and generalise this result to the case of a single one.

In the next section, we discuss the generation of one-loop numerators from the Jacobi off-shell currents of section 3. In order to do so, we rely on the Loop-Tree duality formalism.

\section{Colour-kinematics duality for $2 \rightarrow 2$ processes at one-loop}

In this section, we study the one-loop $2 \rightarrow 2$ processes when a numerator is built from the Jacobi off-shell currents (3.5), (3.6) and (3.9). We discuss that at integral level, because of the decomposition shown in section 4 , a new set of relations is generated. In order to see the structure of these relations, we elaborate on the one-loop process of $g g \rightarrow s s$.

\subsection{Integral relations}

At one-loop level we have to deal with objects of this form,

$$
I_{m}=\int_{\ell}\left(\prod_{i=1}^{m} G_{F}\left(q_{i}\right)\right) N\left(\ell,\left\{p_{k}\right\}\right),
$$

where $\int_{\ell}=-\imath \int d^{d} \ell /(2 \pi)^{d}, G_{F}\left(q_{i}\right)=\left(q_{i}^{2}+\imath 0\right)^{-1}$ are massless Feynman propagators, and $N\left(\ell,\left\{p_{k}\right\}\right)$ is the numerator built from the Jacobi off-shell currents $J_{\mathrm{s}, \mathrm{q}, \mathrm{g}}$. The natural study of this object is considering its behaviour when it is embedded in one-loop topologies with two and three internal propagators, as depicted in figure 3.

From figure 3 we note that at one-loop level we have an off-shell current attached to two off- and two on-shell momenta. Hence, we recall the results of section 4.2 for $N_{\mathrm{s}, \mathrm{q}, \mathrm{g}}^{\mu_{1} \ldots \mu_{4}}$, whose decomposition in terms of off-shell momenta allows to provide relations at integral level. It turns out that if the numerator (5.1) belongs to a triangle or bubble diagram, it 
collapses into

$$
\begin{aligned}
& I_{3}=\int_{\ell} \frac{N_{3}}{\ell^{2}\left(\ell+p_{3}\right)^{2}\left(\ell+p_{34}\right)^{2}}
\end{aligned}
$$

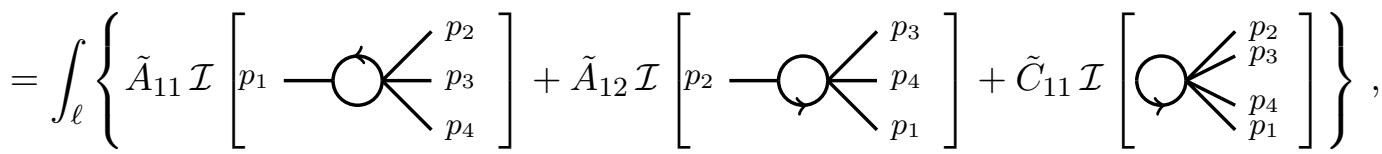

and

$$
\begin{aligned}
& I_{2}=\int_{\ell} \frac{N_{2}}{\ell^{2}\left(\ell+p_{34}\right)^{2}}
\end{aligned}
$$

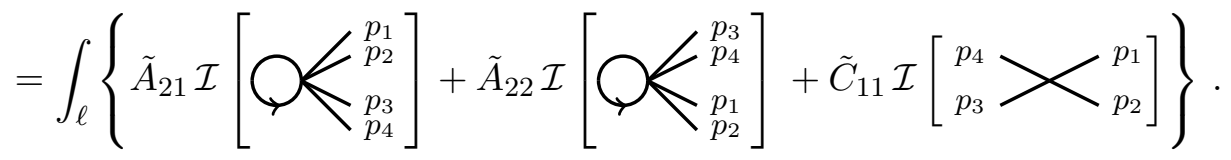

Here, $\mathcal{I}[\cdots]$ states for the integrand of the topology, $\tilde{A}$ and $\tilde{C}$ are polynomials in the loop momentum $\ell$, whose structure follows the results of section 4.2 .

We observe that the r.h.s. of eq. (5.2) corresponds to topologies that integrate to zero in dimensional regularisation, massless bubbles and tadpoles. Therefore, because of the Jacobi numerators (4.8), (4.10) and (4.12), we obtain relations between integrals with the same number of loop propagators, three and two. These relations play a fundamental role when calculating an amplitude through the Loop-Tree duality (LTD) formalism. This is because different combinations of integrals can vanish by means of the outcome of CKD. We remark that apart of performing the single cuts given by the prescriptions of LTD we also integrate out the expressions. It is indeed for this reason that the use of relations (5.2) before integrating increases the efficiency in the evaluation of any amplitude, since terms that seem to have a cumbersome structure at integrand level can be replaced by others holding a simpler structure.

The recent work of ref.s [26, 27] has addressed an alternative study of numerators built from the off-shell current $J_{\mathrm{g}}$. The approach of the authors is based on monodromy relations in string theory. In particular, with the limit $\alpha^{\prime} \rightarrow 0$, the expansion of monodromy relations in terms of cubic diagrams turns out to be a linear combination of Jacobi identities.

For the sake of simplicity, let us recall the results of ref. [27, eq. (4.12)] for $2 \rightarrow 2$ processes, which, in the notation of the current paper, become

$$
\begin{aligned}
& 0=\int_{\ell}\left\{\frac{1}{\ell^{2}\left(\ell+p_{12}\right)^{2}\left(\ell-p_{4}\right)^{2}} n\left(\zeta_{p_{1}}^{p_{2}} \coprod_{p_{4}}^{p_{3}}\right)-\frac{1}{\ell^{2}\left(\ell+p_{2}\right)^{2}\left(\ell+p_{23}\right)^{2}} n(\overbrace{p_{1}}^{p_{4}} \overbrace{p_{3}}^{p_{2}})\right. \\
& +\frac{1}{\ell^{2}\left(\ell+p_{2}\right)^{2}\left(\ell-p_{4}\right)^{2}} n(\overbrace{p_{1}}^{p_{3}} \overbrace{p_{2}}^{p_{4}})+\frac{1}{s_{12} \ell^{2}\left(\ell+p_{12}\right)^{2}} n(\overbrace{p_{1}}^{p_{2}} \\
& \left.-\frac{1}{s_{23}\left(\ell+p_{1}\right)^{2}\left(\ell-p_{4}\right)^{2}} n\left({ }_{p_{1}}^{p_{4}}(J)<p_{p_{3}}^{p_{2}}\right)+\frac{1}{s_{24}\left(\ell+p_{2}\right)^{2}\left(\ell-p_{4}\right)^{2}} n\left(\sum_{p_{1}}^{p_{3}} \nearrow_{p_{2}}^{p_{4}}\right)\right\} \text {. }
\end{aligned}
$$

The $+\imath 0$ prescription of the Feynman propagators is implicitly understood. 
From the diagrammatic and deep study of the Jacobi off-shell currents provided in sections 3 and 4, we observe that the very same behaviour of eq. (5.3) is recovered in decompositions (4.8), (4.10) and (4.12). Moreover, the difference relies on the fact that we know apriori the structure of any object built from the off-shell currents $J_{\mathrm{s}, \mathrm{q}, \mathrm{g}}$. Therefore, we can eliminate topologies that at this point do not contribute and generate redundant contributions. This set of $2 \rightarrow 2$ massless processes do not show any difficulty in the calculation, since grouping integrands according to Jacobi identities satisfies CKD at integral level.

\section{$5.2 \quad g g \rightarrow s s$ at one-loop}

In this section, we provide an application of CKD for one-loop $2 \rightarrow 2$ processes. To this end, we embed the Jacobi off-shell currents in a one-loop topology. Since for this set up two particles are on- and the other two are off-shell, we consider, without loss of generality, the off-shell current $J_{\mathrm{g}}$. This is because the behaviour of the complete numerator $N_{\mathrm{g}}$ will be the same as for $N_{\mathrm{s}}$ and $N_{\mathrm{q}}$ when two of the four particles attached to the Jacobi off-shell current are set on-shell, as shown in section 4.2. This calculation is made in the framework of the LTD.

In order to write numerators as simple as posible we decompose them in terms of form factors by using Lorentz invariance. In fact, the recent work of [49] deals with the Higgs production via gluon fusion, $g g \rightarrow H$ using LTD formalism. We can extend this set up for the current calculation. This is due to the fact that the particles carrying the information of the process are external gluons.

Let us consider a one-loop object

$$
\left|\mathcal{M}_{g g \rightarrow s s}^{(1)}\right\rangle=i g_{s}^{4} \varepsilon^{\mu_{1}}\left(p_{1}\right) \varepsilon^{\mu_{2}}\left(p_{2}\right) \mathcal{A}_{\mu_{1} \mu_{2}}^{(1)},
$$

where $\mathcal{A}$ is built from the Jacobi off-shell current $J_{\mathrm{g}}$. As mentioned above, because of Lorentz invariance, this tensor is given by

$$
\mathcal{A}_{\mu_{1} \mu_{2}}^{(1)}=\sum_{i=1}^{10} A_{i}^{(1)} T_{\mu_{1} \mu_{2}}^{i},
$$

with $A_{i}$ the form factors or scalar functions of the Maldestam variables $s_{12}, s_{23}$ and of the dimension $d$, and $T$ the tensor basis

$$
\begin{aligned}
T_{i}^{\mu_{1} \mu_{2}}=\{ & g^{\mu_{1} \mu_{2}}-\frac{2 p_{1}^{\mu_{2}} p_{2}^{\mu_{1}}}{s_{12}}, g^{\mu_{1} \mu_{2}}, \frac{2 p_{1}^{\mu_{2}} p_{3}^{\mu_{1}}}{s_{13}}, \frac{2 p_{2}^{\mu_{1}} p_{3}^{\mu_{2}}}{s_{23}}, \frac{2 s_{12} p_{3}^{\mu_{1}} p_{3}^{\mu_{2}}}{s_{23} s_{13}}, \\
& \left.\frac{2 p_{1}^{\mu_{1}} p_{2}^{\mu_{2}}}{s_{12}}, \frac{2 p_{1}^{\mu_{1}} p_{1}^{\mu_{2}}}{s_{12}}, \frac{2 p_{2}^{\mu_{1}} p_{2}^{\mu_{2}}}{s_{12}}, \frac{2 p_{1}^{\mu_{1}} p_{3}^{\mu_{2}}}{s_{23}}, \frac{2 p_{2}^{\mu_{2}} p_{3}^{\mu_{1}}}{s_{13}}\right\},
\end{aligned}
$$

with $s_{13}=-s_{12}-s_{23}$.

Since external gluons obey the transversality condition, $p_{i} \cdot \varepsilon\left(p_{i}\right)=0$, we only compute the first five contributions of (5.5). These scalar coefficients are extracted by applying appropriate projecting operators on the tensor (5.5), such that $P_{i}^{\mu \nu} \mathcal{A}_{\mu \nu}^{(1)}=A_{i}^{(1)}$. All projectors are collected in appendix B. 
In section 4.2 we studied the general case of the four off-shell legs attached to the Jacobi off-shell currents. For the one-loop case we are considering, with two off-shell particles, the parametric decomposition (4.12) takes the form,

$$
N_{g}=c_{2}\left(\ell+p_{34}\right)^{2}+c_{3} \ell^{2}+c_{23} \ell^{2}\left(\ell+p_{34}\right)^{2},
$$

with $c$ scalar coefficients depending on the external and internal momenta, and $\ell$ the internal loop momentum. Since this numerator is sat either in two or three loop massless propagators, we can independently elaborate on each coefficient. This is done because each coefficient together with the set of propagators vanishes in dimensional regularisation as depicted in eq. (5.2).

In order to explain how integral relations are obtained, let us first consider the case when the numerator (5.7) is sat in three loop propagators (see figure 3.a). We find, for the form factor $A_{1}$ two independent identities, ${ }^{1}$

$$
\begin{aligned}
& \int_{\ell} \frac{c_{2}^{(1)}\left(\ell+p_{34}\right)^{2}}{\ell^{2}\left(\ell+p_{3}\right)^{2}\left(\ell+p_{34}\right)^{2}}=\int_{\ell}\left\{\delta ( k _ { 1 } ) \frac { ( 2 k _ { 1 } \cdot p _ { 1 } + s _ { 2 3 } ) } { ( 2 k _ { 1 } \cdot p _ { 4 } ) } \left[( k _ { 1 } \cdot p _ { 1 } ) \left(-\left(s_{12}^{2}+s_{12} s_{23}-s_{23}^{2}\right)\left(k_{1} \cdot p_{3}\right)\right.\right.\right. \\
& \left.+s_{23}\left(s_{12}^{2}+4 s_{12} s_{23}+2 s_{23}^{2}-s_{13}\left(k_{1} \cdot p_{4}\right)\right)+s_{12} s_{13}\left(k_{1} \cdot p_{2}\right)\right) \\
& +s_{13}^{2}\left(k_{1} \cdot p_{2}\right)\left(k_{1} \cdot p_{34}+2 s_{23}\right)-s_{12} s_{23}\left(k_{1} \cdot p_{1}\right)^{2} \\
& \left.+s_{12}\left(-s_{13}\left(k_{1} \cdot p_{3}\right)^{2}+\left(k_{1} \cdot p_{3}\right)\left(-s_{13}\left(k_{1} \cdot p_{4}\right)+s_{23}\left(3 s_{12}+4 s_{23}\right)\right)-s_{23} s_{13}\left(k_{1} \cdot p_{4}+s_{23}\right)\right)\right] \\
& -\delta\left(k_{3}\right) \frac{\left(k_{3} \cdot p_{1}+s_{23}\right)}{\left(2 k_{3} \cdot p_{4}\right)}\left[( k _ { 3 } \cdot p _ { 1 } ) \left(-2\left(s_{12}^{2}+s_{12} s_{23}-s_{23}^{2}\right)\left(k_{3} \cdot p_{3}\right)\right.\right. \\
& \left.+2 s_{12} s_{13}\left(k_{3} \cdot p_{2}\right)+2 s_{23} s_{13}\left(k_{3} \cdot p_{4}\right)+s_{23}\left(s_{12}+2 s_{23}\right)\left(3 s_{12}+2 s_{23}\right)\right) \\
& \left.\left.-2 s_{12} s_{23}\left(k_{3} \cdot p_{1}\right)^{2}+\left(-s_{13}\left(k_{3} \cdot p_{2}\right)+s_{12}\left(k_{3} \cdot p_{3}\right)\right)\left(-2 s_{13} k_{3} \cdot\left(p_{3}-p_{4}\right)+\left(s_{12}+2 s_{23}\right)^{2}\right)\right]\right\}=0, \\
& \int_{\ell} \frac{c_{3}^{(1)} \ell^{2}}{\ell^{2}\left(\ell+p_{3}\right)^{2}\left(\ell+p_{34}\right)^{2}}=\int_{\ell}\left\{\frac { \tilde { \delta } ( k _ { 1 } ) ( 2 k _ { 1 } \cdot p _ { 1 } - s _ { 1 3 } ) } { 8 ( 2 k _ { 1 } \cdot p _ { 3 } ) } \left[( k _ { 1 } \cdot p _ { 1 } ) \left(2 s_{12} s_{13}\left(k_{1} \cdot p_{2}\right)+2 s_{23} s_{13}\left(k_{1} \cdot p_{4}\right)\right.\right.\right. \\
& \left.-2\left(s_{12}^{2}+3 s_{23} s_{12}+s_{23}^{2}\right)\left(k_{1} \cdot p_{3}\right)-s_{23}\left(s_{12}+2 s_{23}\right)\left(3 s_{12}+2 s_{23}\right)\right) \\
& -2 s_{12} s_{23}\left(k_{1} \cdot p_{1}\right)^{2}+s_{13}\left(k_{1} \cdot p_{2}\right)\left(\left(s_{12}+2 s_{23}\right)^{2}-2 s_{13}\left(k_{1} \cdot p_{3}+k_{1} \cdot p_{4}\right)\right) \\
& \left.+s_{12}\left(-\left(k_{1} \cdot p_{3}\right)\left(-2 s_{13} k_{1} \cdot p_{34}+s_{12}^{2}+2 s_{23} s_{12}+2 s_{23}^{2}\right)-s_{13} s_{23}\left(s_{12}+2 s_{23}\right)\right)\right] \\
& +\frac{\tilde{\delta}\left(k_{2}\right)\left(k_{2} \cdot p_{1}-s_{13}\right)}{4\left(2 k_{2} \cdot p_{3}\right)}\left[( k _ { 2 } \cdot p _ { 1 } ) \left(-2 s_{12} s_{13}\left(k_{2} \cdot p_{2}\right)-2 s_{23} s_{13}\left(k_{2} \cdot p_{4}\right)\right.\right. \\
& \left.+2\left(s_{12}^{2}+s_{23} s_{12}-s_{23}^{2}\right)\left(k_{2} \cdot p_{3}\right)+s_{23}\left(s_{12}+2 s_{23}\right)\left(3 s_{12}+2 s_{23}\right)\right) \\
& \left.\left.+2 s_{12} s_{23}\left(k_{2} \cdot p_{1}\right)^{2}+\left(s_{12}\left(k_{2} \cdot p_{3}\right)-s_{13}\left(k_{2} \cdot p_{2}\right)\right)\left(2 s_{13} k_{2} \cdot\left(p_{3}-k_{2}\right)+\left(s_{12}+2 s_{23}\right)^{2}\right)\right]\right\}=0,
\end{aligned}
$$

${ }^{1}$ The scalar coefficients $c_{i}^{(j)}$ follow the structure of the ones of eq. (5.7) and account for the information of the projectors $P_{j}^{\mu \nu}$ of eq. (B.1). 
Terms proportional to $\ell^{2}\left(\ell+p_{34}\right)^{2}$ do not appear in this analysis.

Similarly for $A_{2}$,

$$
\begin{aligned}
& \int_{\ell} \frac{c_{2}^{(2)}\left(\ell+p_{34}\right)^{2}}{\ell^{2}\left(\ell+p_{3}\right)^{2}\left(\ell+p_{34}\right)^{2}}=\int_{\ell}\left\{\frac { \tilde { \delta } ( k _ { 1 } ) ( 2 ( k _ { 1 } \cdot p _ { 1 } ) + s _ { 2 3 } ) } { ( 2 k _ { 1 } \cdot p _ { 4 } ) } \left[( k _ { 1 } \cdot p _ { 1 } ) \left(-s_{13} s_{23}\left(k_{1} \cdot p_{4}\right)+s_{12} s_{13}\left(k_{1} \cdot p_{2}\right)\right.\right.\right. \\
& \left.-\left(s_{12}^{2}+s_{23} s_{12}-s_{23}^{2}\right)\left(k_{1} \cdot p_{3}\right)+s_{12} s_{23}^{2}\right) \\
& -s_{12} s_{23}\left(k_{1} \cdot p_{1}\right)^{2}-s_{13}\left(k_{1} \cdot p_{2}\right)\left(s_{12} s_{23}-s_{13}\left(k_{1} \cdot p_{34}\right)\right) \\
& \left.+s_{12}\left(-s_{13}\left(k_{1} \cdot p_{4}\right)\left(k_{1} \cdot p_{3}+2 s_{23}\right)+\left(k_{1} \cdot p_{3}\right)\left(s_{23}\left(4 s_{12}+5 s_{23}\right)-s_{13}\left(k_{1} \cdot p_{3}\right)\right)+s_{12} s_{13} s_{23}\right)\right] \\
& +\frac{\tilde{\delta}\left(k_{3}\right)\left(k_{3} \cdot p_{1}+s_{23}\right)}{\left(2 k_{3} \cdot p_{4}\right)}\left[( k _ { 3 } \cdot p _ { 1 } ) \left(2 s_{12} s_{13}\left(k_{3} \cdot p_{2}\right)+2 s_{23} s_{13}\left(k_{3} \cdot p_{4}\right)\right.\right. \\
& \left.-2\left(s_{12}^{2}+s_{23} s_{12}-s_{23}^{2}\right)\left(k_{3} \cdot p_{3}\right)+s_{12} s_{23}\left(s_{12}+2 s_{23}\right)\right) \\
& +2 s_{12} s_{13}\left(k_{3} \cdot p_{4}\right)\left(k_{3} \cdot p_{3}+s_{23}\right)+s_{12}\left(k_{3} \cdot p_{3}\right)\left(-2 s_{13}\left(k_{3} \cdot p_{3}\right)+s_{12}^{2}+6 s_{23} s_{12}+6 s_{23}^{2}\right) \\
& \left.\left.-2 s_{12} s_{23}\left(k_{3} \cdot p_{1}\right)^{2}-s_{13}\left(k_{3} \cdot p_{2}\right)\left(s_{12}\left(s_{12}+2 s_{23}\right)-2 s_{13} k_{3} \cdot\left(p_{3}-p_{4}\right)\right)\right]\right\}=0 \text {, } \\
& \int_{\ell} \frac{c_{3}^{(2)} \ell^{2}}{\ell^{2}\left(\ell+p_{3}\right)^{2}\left(\ell+p_{34}\right)^{2}}=\int_{\ell}\left\{\frac { \tilde { \delta } ( k _ { 1 } ) ( 2 ( k _ { 1 } \cdot p _ { 1 } ) - s _ { 1 3 } ) } { 2 ( 2 k _ { 1 } \cdot p _ { 3 } ) } \left[( k _ { 1 } \cdot p _ { 1 } ) \left(2 s_{12} s_{13}\left(k_{1} \cdot p_{2}\right)+2 s_{23} s_{13}\left(k_{1} \cdot p_{4}\right)\right.\right.\right. \\
& \left.-2\left(s_{12}^{2}+3 s_{23} s_{12}+s_{23}^{2}\right)\left(k_{1} \cdot p_{3}\right)-s_{12} s_{23}\left(s_{12}+2 s_{23}\right)\right) \\
& +s_{12}\left(2 s_{13}\left(k_{1} \cdot p_{3}\right)^{2}-\left(k_{1} \cdot p_{3}\right)\left(s_{12}^{2}-2 s_{13}\left(k_{1} \cdot p_{4}\right)\right)-s_{13} s_{23}\left(2\left(k_{1} \cdot p_{4}\right)+s_{12}\right)\right) \\
& \left.-2 s_{12} s_{23}\left(k_{1} \cdot p_{1}\right)^{2}+s_{13}\left(k_{1} \cdot p_{2}\right)\left(s_{12}\left(s_{12}+2 s_{23}\right)-2 s_{13}\left(k_{1} \cdot p_{3}+k_{1} \cdot p_{4}\right)\right)\right] \\
& +\frac{\delta\left(k_{2}\right)\left(k_{2} \cdot p_{1}-s_{13}\right)}{\left(2 k_{2} \cdot p_{3}\right)}\left[( k _ { 2 } \cdot p _ { 1 } ) \left(-2 s_{12} s_{13}\left(k_{2} \cdot p_{2}\right)-2 s_{23} s_{13}\left(k_{2} \cdot p_{4}\right)\right.\right. \\
& \left.+2\left(s_{12}^{2}+s_{23} s_{12}-s_{23}^{2}\right)\left(k_{2} \cdot p_{3}\right)+s_{12} s_{23}\left(s_{12}+2 s_{23}\right)\right) \\
& -2 s_{12} s_{13}\left(k_{2} \cdot p_{4}\right)\left(k_{2} \cdot p_{3}-s_{23}\right)+s_{12}\left(k_{2} \cdot p_{3}\right)\left(2 s_{13}\left(k_{2} \cdot p_{3}\right)+s_{12}^{2}+6 s_{23} s_{12}+6 s_{23}^{2}\right) \\
& \left.\left.+2 s_{12} s_{23}\left(k_{2} \cdot p_{1}\right)^{2}-s_{13}\left(k_{2} \cdot p_{2}\right)\left(2 s_{13}\left(k_{2} \cdot p_{3}-k_{2} \cdot p_{4}\right)+s_{12}\left(s_{12}+2 s_{23}\right)\right)\right]\right\}=0
\end{aligned}
$$

where $\tilde{\delta}\left(k_{i}\right)=2 \pi \imath \delta^{(+)}\left(k_{i}^{2}\right)$ and $k_{i}$ are defined as follows,

$$
k_{1}=\ell+p_{4}, \quad k_{2}=\ell+p_{34}, \quad k_{3}=\ell,
$$

whose on-shell loop energies are given by

$$
k_{1,0}^{(+)}=\sqrt{\left(\boldsymbol{\ell}+\boldsymbol{p}_{\mathbf{4}}\right)^{2}}, \quad k_{2,0}^{(+)}=\sqrt{\left(\boldsymbol{\ell}+\boldsymbol{p}_{\mathbf{3 4}}\right)^{2}}, \quad k_{3,0}^{(+)}=\sqrt{\boldsymbol{\ell}^{2}}, .
$$


In eq.s (5.8) and (5.9) we observe relations between integrals that appear from different cuts. Nevertheless, for these $2 \rightarrow 2$ processes it is convenient to work in the center-of-mass frame, because of the additional constraint $k_{2,0}^{(+)}=k_{3,0}^{(+)}=\ell_{0}^{(+)}$, which turns out to be equivalent to $\tilde{\delta}\left(k_{2}\right)=\tilde{\delta}\left(k_{3}\right)=\tilde{\delta}(\ell)$. This constraint allows us to combine both results, for instance the two identities in eq.s (5.8) or (5.9), to express the integrals that depend on $\tilde{\delta}\left(k_{1}\right)$ in terms of simpler ones.

A similar study, even though it is not that illuminating for the purpose of this example, can be done for the other form factors, $A_{i}$, with $i=3,4,5$, as well as for the case of the numerator (5.7) with two loop propagators, which is depicted in figure 3.b. The difference with the above example relies on the rank of the numerators, since for this one we obtain a polynomial in $\ell$ with at most rank two. However, in order to get further simplifications in the integral relations, contributions of 3.b do have to be taken into account.

\section{Conclusions}

In this paper, we have studied the colour-kinematics duality (CKD) at tree and one-loop level. We have computed the off-shell currents in the Feynman and axial gauges from the Jacobi-like combinations of numerators. We have considered the QCD processes $g g \rightarrow$ $s s, q \bar{q}, g g$, finding that the most compact form for these off-shell currents can always be written in terms of three-point interaction Feynman rules. Although, these interactions do not always obey the usual properties of the Feynman rules, they allow for a universal representation in terms of three-point interactions. We have also seen that these off-shell currents vanish if the external particles are set on-shell, satisfying CKD.

Consequently, we have embedded the off-shell currents in a richer topology, at tree level with higher-multiplicity or one-loop, obtaining that with a decomposition of the four momenta entering to the off-shell currents in terms of on-shell massless ones, any object built from these currents can be written as a linear combination of at most two of the four squared momenta, $p_{i}^{2} p_{j}^{2}$. This outcome has been achieved as a byproduct of the choice of the gauge, since the reference momentum has chosen to be the same for all internal gluons.

The decomposition of one-loop numerators has been extended, finding relations between integrals with the same number of propagators. For the $2 \rightarrow 2$ case, we have written relations for Feynman integrals with two and three loop propagators. In the same manner and in the framework of the Loop-Tree duality formalism, we have considered the particular example of $g g \rightarrow s s$ showing that higher rank numerators can be replaced by lower ones. This outcome indeed allows for an optimisation in the evaluation of Feynman integrals.

Furthermore, we have observed that monodromy relations obtained in ref. [27] in tandem with the structure of numerators built from the Jacobi off-shell currents play a very important role for finding integral relations for $2 \rightarrow n$ processes with $n>2$. In particular, the linear combination of Jacobi identities in the kinematical sector, provided by monodromy relations, generates all posible integral relations. However, several topologies turn out to be redundant, since the Jacobi identity of kinematic numerators shows the same pattern we have discussed through this paper in which their contribution vanish upon integration. 
Future work could include the consequences of these integral relations at higher loop orders, in particular if the calculation of Integration-by-parts identities [50-52] gets optimised when there is a new ingredient provided by CKD.

\section{Acknowledgments}

This work is supported by the Spanish Government and ERDF funds from European Commission (Grants No. FPA2014-53631-C2-1-P and SEV-2014- 0398) and by Consejo Superior de Investigaciones Científicas (Grant No. PIE-201750E021). The work of J.L. has been supported by Instituto de Física Corpuscular.

\section{A Three-point interaction Feynman rules}

In this appendix, we collect the colour stripped Feynman rules of section 2,

$$
\underbrace{p_{2}}_{\bar{p}_{3}}=\left(p_{2}-p_{3}\right)^{\mu_{1}}
$$

\section{B Projectors}

In this appendix, we collect the projectors used to extract the form factors $A_{i}$ of section 5.2

$$
\begin{aligned}
P_{1}^{\mu_{1} \mu_{2}=}= & \frac{1}{2(d-3)}\left[g^{\mu_{1} \mu_{2}}-\frac{(d-2) p_{1}^{\mu_{2}} p_{2}^{\mu_{1}}}{s_{12}}+\frac{(d-4) p_{3}^{\mu_{2}} p_{2}^{\mu_{1}}}{s_{23}}+\frac{(d-2) s_{13} p_{2}^{\mu_{2}} p_{2}^{\mu_{1}}}{s_{12} s_{23}}+\frac{(d-2) s_{23} p_{1}^{\mu_{1}} p_{1}^{\mu_{2}}}{s_{12} s_{13}}\right. \\
& \left.-\frac{(d-2) p_{1}^{\mu_{1}} p_{2}^{\mu_{2}}}{s_{12}}+\frac{(d-4) p_{1}^{\mu_{2}} p_{3}^{\mu_{1}}}{s_{13}}-\frac{(d-2) p_{1}^{\mu_{1}} p_{3}^{\mu_{2}}}{s_{13}}-\frac{(d-2) p_{2}^{\mu_{2}} p_{3}^{\mu_{1}}}{s_{23}}-\frac{(d-4) s_{12} p_{3}^{\mu_{1}} p_{3}^{\mu_{2}}}{s_{13} s_{23}}\right], \\
P_{2}^{\mu_{1} \mu_{2}=} & \frac{1}{2(d-3)}\left[g^{\mu_{1} \mu_{2}}+\frac{(d-4) p_{1}^{\mu_{2}} p_{2}^{\mu_{1}}}{s_{12}}-\frac{(d-2) p_{3}^{\mu_{2}} p_{2}^{\mu_{1}}}{s_{23}}-\frac{(d-4) s_{13} p_{2}^{\mu_{2}} p_{2}^{\mu_{1}}}{s_{12} s_{23}}-\frac{(d-4) s_{23} p_{1}^{\mu_{1}} p_{1}^{\mu_{2}}}{s_{12} s_{13}}\right. \\
& \left.+\frac{(d-4) p_{1}^{\mu_{1}} p_{2}^{\mu_{2}}}{s_{12}}-\frac{(d-2) p_{1}^{\mu_{2}} p_{3}^{\mu_{1}}}{s_{13}}+\frac{(d-4) p_{1}^{\mu_{1}} p_{3}^{\mu_{2}}}{s_{13}}+\frac{(d-4) p_{2}^{\mu_{2}} p_{3}^{\mu_{1}}}{s_{23}}+\frac{(d-2) s_{12} p_{3}^{\mu_{1}} p_{3}^{\mu_{2}}}{s_{13} s_{23}}\right],
\end{aligned}
$$




$$
\begin{aligned}
P_{3}^{\mu_{1} \mu_{2}}=\frac{1}{(d-3)}\left[g^{\mu_{1} \mu_{2}}-\frac{(d-2) p_{1}^{\mu_{2}} p_{2}^{\mu_{1}}}{s_{12}}-\frac{(d-2) p_{3}^{\mu_{2}} p_{2}^{\mu_{1}}}{s_{23}}+\frac{(d-2) s_{13} p_{2}^{\mu_{2}} p_{2}^{\mu_{1}}}{s_{12} s_{23}}+\frac{(d-2) s_{23} p_{1}^{\mu_{1}} p_{1}^{\mu_{2}}}{s_{12} s_{13}}\right. & \left.-\frac{(d-2) p_{1}^{\mu_{1}} p_{2}^{\mu_{2}}}{s_{12}}-\frac{(d-2) p_{1}^{\mu_{2}} p_{3}^{\mu_{1}}}{s_{13}}+\frac{(d-4) p_{1}^{\mu_{1}} p_{3}^{\mu_{2}}}{s_{13}}+\frac{(d-4) p_{2}^{\mu_{2}} p_{3}^{\mu_{1}}}{s_{23}}-\frac{(d-4) s_{12} p_{3}^{\mu_{1}} p_{3}^{\mu_{2}}}{s_{13} s_{23}}\right], \\
P_{4}^{\mu_{1} \mu_{2}}=- & \frac{1}{(d-3)}\left[g^{\mu_{1} \mu_{2}}-\frac{(d-2) p_{1}^{\mu_{2}} p_{2}^{\mu_{1}}}{s_{12}}+\frac{(d-4) p_{3}^{\mu_{2}} p_{2}^{\mu_{1}}}{s_{23}}+\frac{(d-2) s_{13} p_{2}^{\mu_{2}} p_{2}^{\mu_{1}}}{s_{12} s_{23}}+\frac{(d-2) s_{23} p_{1}^{\mu_{1}} p_{1}^{\mu_{2}}}{s_{12} s_{13}}\right. \\
& \left.-\frac{(d-2) p_{1}^{\mu_{1}} p_{2}^{\mu_{2}}}{s_{12}}-\frac{(d-2) p_{1}^{\mu_{2}} p_{3}^{\mu_{1}}}{s_{13}}-\frac{(d-2) p_{1}^{\mu_{1}} p_{3}^{\mu_{2}}}{s_{13}}+\frac{(d-4) p_{2}^{\mu_{2}} p_{3}^{\mu_{1}}}{s_{23}}+\frac{(d-2) s_{12} p_{3}^{\mu_{1}} p_{3}^{\mu_{2}}}{s_{13} s_{23}}\right], \\
P_{5}^{\mu_{1} \mu_{2}}= & \frac{1}{(d-3)}\left[g^{\mu_{1} \mu_{2}}-\frac{(d-2) p_{1}^{\mu_{2}} p_{2}^{\mu_{1}}}{s_{12}}-\frac{(d-2) p_{3}^{\mu_{2}} p_{2}^{\mu_{1}}}{s_{23}}+\frac{(d-2) s_{13} p_{2}^{\mu_{2}} p_{2}^{\mu_{1}}}{s_{12} s_{23}}+\frac{(d-2) s_{23} p_{1}^{\mu_{1}} p_{1}^{\mu_{2}}}{s_{12} s_{13}}\right. \\
& \left.-\frac{(d-2) p_{1}^{\mu_{1}} p_{2}^{\mu_{2}}}{s_{12}}+\frac{(d-4) p_{1}^{\mu_{2}} p_{3}^{\mu_{1}}}{s_{13}}+\frac{(d-4) p_{1}^{\mu_{1}} p_{3}^{\mu_{2}}}{s_{13}}-\frac{(d-2) p_{2}^{\mu_{2}} p_{3}^{\mu_{1}}}{s_{23}}+\frac{(d-2) s_{12} p_{3}^{\mu_{1}} p_{3}^{\mu_{2}}}{s_{13} s_{23}}\right] .
\end{aligned}
$$

Open Access. This article is distributed under the terms of the Creative Commons Attribution License (CC-BY 4.0), which permits any use, distribution and reproduction in any medium, provided the original author(s) and source are credited.

\section{References}

[1] P. Cvitanovic, P.G. Lauwers and P.N. Scharbach, Gauge Invariance Structure of Quantum Chromodynamics, Nucl. Phys. B 186 (1981) 165 [INSPIRE].

[2] F.A. Berends and W. Giele, The Six Gluon Process as an Example of Weyl-Van Der Waerden Spinor Calculus, Nucl. Phys. B 294 (1987) 700 [INSPIRE].

[3] M.L. Mangano, S.J. Parke and Z. Xu, Duality and Multi-Gluon Scattering, Nucl. Phys. B 298 (1988) 653 [inSPIRE].

[4] D. Kosower, B.-H. Lee and V.P. Nair, Multi Gluon Scattering: A String Based Calculation, Phys. Lett. B 201 (1988) 85 [InSPIRE].

[5] Z. Bern and D.A. Kosower, Color decomposition of one loop amplitudes in gauge theories, Nucl. Phys. B 362 (1991) 389 [inSPIRE].

[6] V. Del Duca, L.J. Dixon and F. Maltoni, New color decompositions for gauge amplitudes at tree and loop level, Nucl. Phys. B 571 (2000) 51 [hep-ph/9910563] [INSPIRE].

[7] F. Maltoni, K. Paul, T. Stelzer and S. Willenbrock, Color flow decomposition of QCD amplitudes, Phys. Rev. D 67 (2003) 014026 [hep-ph/0209271] [InSPIRE].

[8] R. Kleiss and H. Kuijf, Multi-Gluon Cross-sections and Five Jet Production at Hadron Colliders, Nucl. Phys. B 312 (1989) 616 [inSPIRE].

[9] Z. Bern, J.J.M. Carrasco and H. Johansson, New Relations for Gauge-Theory Amplitudes, Phys. Rev. D 78 (2008) 085011 [arXiv:0805.3993] [INSPIRE]. 
[10] Z. Bern, J.J.M. Carrasco and H. Johansson, Perturbative Quantum Gravity as a Double Copy of Gauge Theory, Phys. Rev. Lett. 105 (2010) 061602 [arXiv:1004.0476] [INSPIRE].

[11] Z. Bern, J.J.M. Carrasco, L.J. Dixon, H. Johansson and R. Roiban, Simplifying Multiloop Integrands and Ultraviolet Divergences of Gauge Theory and Gravity Amplitudes, Phys. Rev. D 85 (2012) 105014 [arXiv:1201.5366] [inSPIRE].

[12] J.J. Carrasco and H. Johansson, Five-Point Amplitudes in $N=4$ super-Yang-Mills Theory and $N=8$ Supergravity, Phys. Rev. D 85 (2012) 025006 [arXiv:1106.4711] [INSPIRE].

[13] N.E.J. Bjerrum-Bohr, T. Dennen, R. Monteiro and D. O'Connell, Integrand Oxidation and One-Loop Colour-Dual Numerators in N=4 Gauge Theory, JHEP 07 (2013) 092 [arXiv: 1303.2913] [INSPIRE].

[14] J.J.M. Carrasco, M. Chiodaroli, M. Günaydin and R. Roiban, One-loop four-point amplitudes in pure and matter-coupled $N \leq 4$ supergravity, JHEP 03 (2013) 056 [arXiv: 1212.1146] [INSPIRE].

[15] Z. Bern, C. Boucher-Veronneau and H. Johansson, $N \geq 4$ Supergravity Amplitudes from Gauge Theory at One Loop, Phys. Rev. D 84 (2011) 105035 [arXiv:1107.1935] [InSPIRE].

[16] C. Boucher-Veronneau and L.J. Dixon, $N \geq 4$ Supergravity Amplitudes from Gauge Theory at Two Loops, JHEP 12 (2011) 046 [arXiv:1110.1132] [INSPIRE].

[17] P. Mastrolia, E. Mirabella, G. Ossola and T. Peraro, Integrand-Reduction for Two-Loop Scattering Amplitudes through Multivariate Polynomial Division, Phys. Rev. D 87 (2013) 085026 [arXiv:1209.4319] [INSPIRE].

[18] S. He, R. Monteiro and O. Schlotterer, String-inspired BCJ numerators for one-loop MHV amplitudes, JHEP 01 (2016) 171 [arXiv:1507.06288] [INSPIRE].

[19] G. Yang, Color-kinematics duality and Sudakov form factor at five loops for $N=4$ supersymmetric Yang-Mills theory, Phys. Rev. Lett. 117 (2016) 271602 [arXiv:1610.02394] [INSPIRE].

[20] Z. Bern, J.J.M. Carrasco, W.-M. Chen, H. Johansson, R. Roiban and M. Zeng, The Five-Loop Four-Point Integrand of $N=8$ Supergravity as a Generalized Double Copy, arXiv:1708.06807 [INSPIRE].

[21] Z. Bern, S. Davies, T. Dennen, Y.-t. Huang and J. Nohle, Color-Kinematics Duality for Pure Yang-Mills and Gravity at One and Two Loops, Phys. Rev. D 92 (2015) 045041 [arXiv: 1303.6605] [INSPIRE].

[22] J. Nohle, Color-Kinematics Duality in One-Loop Four-Gluon Amplitudes with Matter, Phys. Rev. D 90 (2014) 025020 [arXiv:1309.7416] [INSPIRE].

[23] S. Badger, G. Mogull, A. Ochirov and D. O'Connell, A Complete Two-Loop, Five-Gluon Helicity Amplitude in Yang-Mills Theory, JHEP 10 (2015) 064 [arXiv:1507.08797] [INSPIRE].

[24] N.E.J. Bjerrum-Bohr, P.H. Damgaard, T. Sondergaard and P. Vanhove, Monodromy and Jacobi-like Relations for Color-Ordered Amplitudes, JHEP 06 (2010) 003 [arXiv:1003.2403] [INSPIRE].

[25] C.R. Mafra, O. Schlotterer and S. Stieberger, Explicit BCJ Numerators from Pure Spinors, JHEP 07 (2011) 092 [arXiv: 1104.5224] [INSPIRE]. 
[26] P. Tourkine and P. Vanhove, Higher-loop amplitude monodromy relations in string and gauge theory, Phys. Rev. Lett. 117 (2016) 211601 [arXiv:1608.01665] [INSPIRE].

[27] A. Ochirov, P. Tourkine and P. Vanhove, One-loop monodromy relations on single cuts, JHEP 10 (2017) 105 [arXiv:1707.05775] [INSPIRE].

[28] S. He, O. Schlotterer and Y. Zhang, New BCJ representations for one-loop amplitudes in gauge theories and gravity, arXiv:1706.00640 [INSPIRE].

[29] D. Chester, Bern-Carrasco-Johansson relations for one-loop QCD integral coefficients, Phys. Rev. D 93 (2016) 065047 [arXiv:1601.00235] [InSPIRE].

[30] A. Primo and W.J. Torres Bobadilla, BCJ Identities and d-Dimensional Generalized Unitarity, JHEP 04 (2016) 125 [arXiv: 1602.03161] [INSPIRE].

[31] P. Mastrolia, A. Primo, U. Schubert and W.J. Torres Bobadilla, Off-shell currents and color-kinematics duality, Phys. Lett. B 753 (2016) 242 [arXiv: 1507.07532] [INSPIRE].

[32] Z. Bern, T. Dennen, Y.-t. Huang and M. Kiermaier, Gravity as the Square of Gauge Theory, Phys. Rev. D 82 (2010) 065003 [arXiv: 1004.0693] [InSPIRE].

[33] N.E.J. Bjerrum-Bohr, P.H. Damgaard, R. Monteiro and D. O'Connell, Algebras for Amplitudes, JHEP 06 (2012) 061 [arXiv:1203.0944] [inSPIRE].

[34] R.H. Boels and R.S. Isermann, On powercounting in perturbative quantum gravity theories through color-kinematic duality, JHEP 06 (2013) 017 [arXiv:1212.3473] [INSPIRE].

[35] S. Catani, T. Gleisberg, F. Krauss, G. Rodrigo and J.-C. Winter, From loops to trees by-passing Feynman's theorem, JHEP 09 (2008) 065 [arXiv: 0804.3170] [INSPIRE].

[36] G.F.R. Sborlini, F. Driencourt-Mangin and G. Rodrigo, Four-dimensional unsubtraction with massive particles, JHEP 10 (2016) 162 [arXiv:1608.01584] [INSPIRE].

[37] G.F.R. Sborlini, F. Driencourt-Mangin, R. Hernandez-Pinto and G. Rodrigo, Four-dimensional unsubtraction from the loop-tree duality, JHEP 08 (2016) 160 [arXiv: 1604.06699] [INSPIRE].

[38] R.J. Hernandez-Pinto, G.F.R. Sborlini and G. Rodrigo, Towards gauge theories in four dimensions, JHEP 02 (2016) 044 [arXiv: 1506. 04617] [INSPIRE].

[39] S. Buchta, G. Chachamis, P. Draggiotis and G. Rodrigo, Numerical implementation of the loop-tree duality method, Eur. Phys. J. C 77 (2017) 274 [arXiv:1510.00187] [INSPIRE].

[40] T. Hahn, Generating Feynman diagrams and amplitudes with FeynArts 3, Comput. Phys. Commun. 140 (2001) 418 [hep-ph/0012260] [INSPIRE].

[41] R. Mertig, M. Böhm and A. Denner, FEYN CALC: Computer algebraic calculation of Feynman amplitudes, Comput. Phys. Commun. 64 (1991) 345 [INSPIRE].

[42] V. Shtabovenko, R. Mertig and F. Orellana, New Developments in FeynCalc 9.0, Comput. Phys. Commun. 207 (2016) 432 [arXiv:1601.01167] [InSPIRE].

[43] Z. Bern, J.J.M. Carrasco, L.J. Dixon, H. Johansson and R. Roiban, The Complete Four-Loop Four-Point Amplitude in $N=4$ super-Yang-Mills Theory, Phys. Rev. D 82 (2010) 125040 [arXiv: 1008.3327] [INSPIRE].

[44] M. Tolotti and S. Weinzierl, Construction of an effective Yang-Mills Lagrangian with manifest BCJ duality, JHEP 07 (2013) 111 [arXiv:1306.2975] [INSPIRE]. 
[45] T. Melia, Dyck words and multiquark primitive amplitudes, Phys. Rev. D 88 (2013) 014020 [arXiv: 1304.7809] [INSPIRE].

[46] T. Melia, Getting more flavor out of one-flavor QCD, Phys. Rev. D 89 (2014) 074012 [arXiv: 1312.0599] [INSPIRE].

[47] H. Johansson and A. Ochirov, Pure Gravities via Color-Kinematics Duality for Fundamental Matter, JHEP 11 (2015) 046 [arXiv: 1407.4772] [INSPIRE].

[48] H. Johansson and A. Ochirov, Color-Kinematics Duality for QCD Amplitudes, JHEP 01 (2016) 170 [arXiv:1507.00332] [INSPIRE].

[49] F. Driencourt-Mangin, G. Rodrigo and G.F.R. Sborlini, Universal dual amplitudes and asymptotic expansions for $g g \rightarrow H$ and $H \rightarrow \gamma \gamma$ in four dimensions, arXiv:1702.07581 [INSPIRE].

[50] F.V. Tkachov, A Theorem on Analytical Calculability of Four Loop Renormalization Group Functions, Phys. Lett. B 100 (1981) 65 [INSPIRE].

[51] K.G. Chetyrkin and F.V. Tkachov, Integration by Parts: The Algorithm to Calculate $\beta$-functions in 4 Loops, Nucl. Phys. B 192 (1981) 159 [InSPIRE].

[52] S. Laporta, High precision calculation of multiloop Feynman integrals by difference equations, Int. J. Mod. Phys. A 15 (2000) 5087 [hep-ph/0102033] [INSPIRE]. 